Ciencia y tecnología

Science and technology 


\title{
Determinación de los parámetros de proceso y caracterización del puré de aguaymanto
}

\author{
Américo Guevara Pérez, Rosella Málaga Barreda \\ Universidad Nacional Agraria La Molina. Lima, Perú \\ Correos electrónicos: aguevara@lamolina.edu.pe,rosella_malaga@hotmail.com \\ Recibido: 11/3/2013 / Aprobado: 23/5/2013
}

\begin{abstract}
Resumen: Se establecieron los parámetros de proceso para obtener puré de aguaymanto (Physalis peruviana L.). En la estandarización, el puré con $4,5 \%$ de almidón modificado y $25^{\circ}$ Brix obtuvo, sensorialmente, un puntaje equivalente a «me gusta mucho». En el tratamiento térmico a $100{ }^{\circ} \mathrm{C}$, durante 24 minutos, se logró la letalidad requerida, UP (unidades de pasteurización) $=3,2$ minutos. Las características del puré fueron: acidez 5,16 $\pm 0,62$ $\mathrm{g}$ ác. cítrico/100 g b.s., $\mathrm{pH} 3,66 \pm 0,14$, azúcares reductores $29,93 \pm 3,77 \mathrm{~g} / 100$ g b.s., color $\mathrm{L}^{*} 21,18 \pm 4,06$, a* $2,59 \pm 1,19, \mathrm{~b}^{*} 16,12 \pm 5.90$, aw $0,973 \pm 0,001 \mathrm{y}$

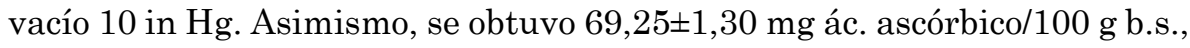
$179,22 \pm 11,94 \mathrm{mg}$ AGE/100 g b.s., 66,25 $\pm 1,44 \mathrm{mg}$ B-caroteno eq./100 g b.s. y 13,62 $\pm 0,75$ mol Trolox eq./ g b.s., al evaluar la vitamina $C$, compuestos fenólicos, carotenoides y capacidad antioxidante, respectivamente.
\end{abstract}

Palabras clave: Pulpeado/ estandarización / tratamiento térmico sensorial / aguaymanto

\section{Golden berry puree process parameters and caracterization}

ABSTRAC: The process parameters to obtain golden berry (Physalis peruviana L.) puree were established. In the standardization, the golden berry puree with $4,5 \%$ and $25^{\circ}$ Brix obtained, sensorially, an "I like it a lot" equivalent score. In the heat treatment test, carried out at $100^{\circ} \mathrm{C}$ during 24 minutes, the requiredlethality wasaccomplished, $\mathrm{PU}$ (pasteurizacion units) $=3,2$ minutes. Puree characteristics were: acidity $5,16 \pm 0,62 \mathrm{~g}$ citric acid/100 g d.b., $\mathrm{pH}$ $3,66 \pm 0,14$, reducing sugars $29,93 \pm 3,77 \mathrm{~g} / 100 \mathrm{~g}$ d.b., color $\mathrm{L}^{*} 21,18 \pm 4,06$, $a^{*} 2,59 \pm 1,19, b^{*} 16,12 \pm 5,90, a_{w} 0,973 \pm 0,001$ and vacuum pressure 10 in $\mathrm{Hg}$. Also, it was obtained $69,25 \pm 1,30 \mathrm{mg}$ ascorbic acid/100 g d.b., 179,22 $\pm 11,94$ mg GAE/100 g d.b., 66,25 $\pm 1,44 \mathrm{mg}$ B-carotene eq./100 g d.b. and 13,62 $\pm 0,75$ $\mu$ mol Trolox eq./ g d.b., of vitamin C, phenolic compounds, carotenoids and antioxidant capacities, respectively.

Keywords: Pulping / standardization / heat treatment sensory / golden berry 


\section{INTRODUCCIÓN}

El consumo de productos procesados a base de frutas es por lo general en forma de jugos, néctares y productos similares, en los cuales las pulpas están considerablemente diluidas, reduciéndose así el aporte nutricional, además de existir el riesgo de producirse enfermedades que transmiten los alimentos (ETA) por el consumo directo de frutos que no son manipulados apropiadamente. Por ello, el puré de aguaymanto envasado es un producto seguro y original, de consumo directo (público en general) o como insumo en la elaboración de golosinas, postres, bebidas y comidas.

La industrialización del aguaymanto en forma de puré constituye un aporte al conocimiento científico y tecnológico, porque coloca a disposición del consumidor un alimento seguro y con alto valor nutricional. Pantelidis et al. (2007) encontraron que el aguaymanto contiene compuestos fenólicos, posee capacidad antioxidante y es fuente de vitamina C. Puente et al. (2011) indican que el aguaymanto contiene importantes cantidades de carotenoides. Según Vasco et al. (2008) y Muñoz et al. (2007), el aguaymanto está incluido dentro del grupo de moderada actividad antioxidante. Por ello, se considera importante el consumo de alimentos a base de aguaymanto, cuyos nutrientes contribuirían a la reducción del riesgo de contraer ciertas enfermedades degenerativas.

En el Perú se producen varias frutas que en función de la composición química se encuentran dentro del grupo de alimentos funcionales; una de ellas es el aguaymanto (Araujo, 2009), de la que según el SIICEX (2012), las exportaciones del fruto fresco se han incrementado de 6.9 TM en el 2007 a 59.2 TM en el 2011 (Estados Unidos es el receptor del $40 \%$ de la exportación y Alemania del 20\%). Así, el aumento de la producción y la demanda del aguaymanto indican su creciente valoración como un alimento de aporte benéfico para la salud, además de agradables propiedades sensoriales. Teniendo en cuenta lo expuesto, se decidió llevar a cabo el trabajo de investigación planteando los siguientes objetivos:

- Determinar los parámetros de procesamiento para obtener puré de aguaymanto.

- Caracterizar el producto obtenido. 


\section{MATERIALES Y MÉTODOS}

\subsection{Lugar de ejecución}

El trabajo de investigación se llevó a cabo en la planta piloto de Tecnología de Alimentos y Productos Agropecuarios (TAPA) y en los laboratorios de las instalaciones pertenecientes a la Facultad de Industrias Alimentarias de la Universidad Nacional Agraria La Molina (UNALM).

\subsection{Materiales y equipos}

\subsubsection{Materia prima e insumos}

- Aguaymanto (Physalis peruviana L.) proveniente de la provincia de Cajamarca.

- Almidón modificado Eliane: Fosfato de di-almidón hidroxipropilado (1442).

- Azúcar blanca refinada industrial

- Envases de vidrio de capacidad nominal $156 \mathrm{~cm}^{3}$, con tapa metálica.

\subsubsection{Reactivos}

- Ácido ascórbico 99\%, Sigma-Aldrich

- Ácido gálico 100\%, Sigma-Aldrich

- B-caroteno 99\%, Sigma-Aldrich

- Trolox (6-hydroxy-2,5,7,8-tetramethylchroman-2-carboxilic acid), 97\% Sigma-Aldrich

\subsubsection{Equipos}

- Agitador orbital de rotación circular Velp Wizard

- Balanza analítica digital AND GR-200. 210 g 10 mg e=1 mg d=0,1mg

- Balanza electrónica Adventurer pro AV812. Ohaus Corporation ${ }^{\circledR}$, USA. Capacidad $810 \mathrm{~g}, \mathrm{~d}=0,01 \mathrm{~g}$

- Balanza electrónica modelo Adventurer pro AV4101. Ohaus Corporation ${ }^{\circledR}$, USA. Capacidad $4.100 \mathrm{~g}, \mathrm{~d}=0,1 \mathrm{~g}$

- Balanza Sores AFM. Capacidad máx. 150 kg, mín. 0,4kg d=20 g 
- Baño María Brookfield TC-202

- Baño María con agitación GFL® 1083

- Centrífuga digital Hettich, D-78522

- Colorímetro Minolta®. CR- 400, Japón

- Computadora Compaq Presario CQ-50-103LA

- Congeladora Coldex CH10P

- Cronómetro digital Casio

- Data Trace Temp System ${ }^{\circledR}$

- Espectrofotómetro Unico® UV-2100

- Estufa eléctrica digital Memmert 100-800. Beschickung, Loading model

- Licuadora Oster 250-022-053

- Medidor de actividad de agua Aqua Lab 3TE

- Micrómetro Mitutoyo (0.25 mm, 0.001 mm). № 293-240, Japón

- Micropipetas Brand (de 10 a 100 y de 100 a 1000 ul)

- Potenciómetro Hanna Instruments, modelo HI8424

- Pulpeadora Vulcano (2.9 mm)

- Refinadora Reeves $(0.5 \mathrm{~mm})$

- Refractómetro digital Atago® Pocket PAL-1. 0.53\%. d=0.01

- Refrigerador-congelador Bosh KDN49

- Termómetro Traceable Control Company

- Tubos de centrífuga de $50 \mathrm{ml}$

- Tubos eppendorf 1.5 y $2 \mathrm{ml}$

- Vacuómetro USG US Gauge Edlund

- Viscosímetro rotacional Brookfield RVDV-III Ultra

2.3 Métodos de análisis

\subsubsection{Análisis fisicoquímico}

Se llevaron a cabo los siguientes análisis fisicoquímicos:

- Humedad (g/100g): AOAC 950.27 (2005)

- Cenizas (g/100g): AOAC 940.26 (2005) 
- Proteína (g/100g): AOAC 920.152 (2005)

- Carbohidratos (g/100g): Por diferencia MS-INN (Collazos, 1993)

- Grasa (g/100g): AOAC 948.22 (2005)

- Fibra cruda (g/100g): NTP 205.003 (1980)

- Energía (kcal/100 g): Por cálculo MS-INN (Collazos, 1993)

- Índice de madurez: Relación de los sólidos solubles y la acidez. NTP 203.121 (2007)

- ${ }^{\circ}$ Brix: refractometría. AOAC 931.12 (2005)

- Acidez (\%): Titulación volumétrica. Acidez valorable total. AOAC 942.15 (2005)

- $\mathrm{pH}$ : potenciometría. AOAC 981.12 (2005)

- Azúcares reductores (g/100g): Lane y Eynon. AOAC 923.09 (2005)

- Actividad enzimática. Peroxidasa (ausencia/presencia): método descrito por Masure y Cambell (1944)

- Actividad de agua: sensores de punto de rocío. Lab-Ferrer. Decagon Devices, Inc.

- Vacío: Medido con un vacuómetro según la NTP 203.077:1977, revisión 2012

- Color: Medición de coordenadas de color según CIE (1986) L* a* b*

- Viscosidad: Jansen et al. (1981) y Marquis et al. (1993), citados por Mouquet y Tréche (2001)

- Vitamina C (mg/100g): método adaptado de AOAC 967.21 (2005)

- Compuestos fenólicos totales (mg AGE/ 100g): método adaptado del reportado por Swain e Hillis (1959)

- Carotenoides (mg B-caroteno eq./ 100g): método adaptado del reportado por Talcott y Howard (1999)

- Capacidad antioxidante ABTS ( $\mathrm{mmol}$ Trolox eq./g): método adaptado del reportado por Arnao, Cano y Acosta (2001).

\subsubsection{Análisis microbiológico}

Se analizaron mohos y levaduras ICMSF (2000) y bacterias ácido-lácticas APHA/CMMEF (2001). 


\subsubsection{Análisis sensorial}

El análisis sensorial fue realizado en la etapa de estandarización, utilizando la metodología para pruebas afectivas de medición del grado de satisfacción, por medio de una escala hedónica verbal de siete puntos, la cual proporciona información medible acerca de la aceptación del producto, mediante descripciones verbales (valoradas) de las sensaciones que producen las muestras a los jueces (Anzaldúa-Morales, 1994). Debido a que el producto sería destinado al consumo de público en general (consumidores potenciales), para lograr confiabilidad en los resultados se requería que el análisis sensorial fuera realizado por 30 (Anzaldúa-Morales, 1994) a 100 (Espinoza, 2003) jueces no entrenados. En una primera etapa, 122 jueces evaluaron 4 muestras codificadas aleatoriamente y en una segunda etapa 90 jueces evaluaron 1 muestra. A los jueces se les solicitó evaluar las muestras (20 gramos de puré) e indicar en la escala el nivel de aceptación general, siguiendo las instrucciones de enjuagarse la boca con agua de mesa antes de probar las muestras.

\subsubsection{Análisis estadístico}

Para evaluar los resultados del análisis sensorial (primera etapa) y establecer la mejor formulación, se determinaron las diferencias estadísticamente significativas entre las medias en base a la prueba de Friedman. Se utilizó el programa Statgraphys Centurion XV.II.

\subsubsection{Flujo de proceso}

En el diagrama 1 se muestra el proceso establecido para la obtención de puré de aguaymanto, cuyo rendimiento, con respecto a los frutos frescos con cáliz, fue de $72 \pm 4 \%$. 


\section{Diagrama 1}

Flujo de operaciones para obtener puré de aguaymanto

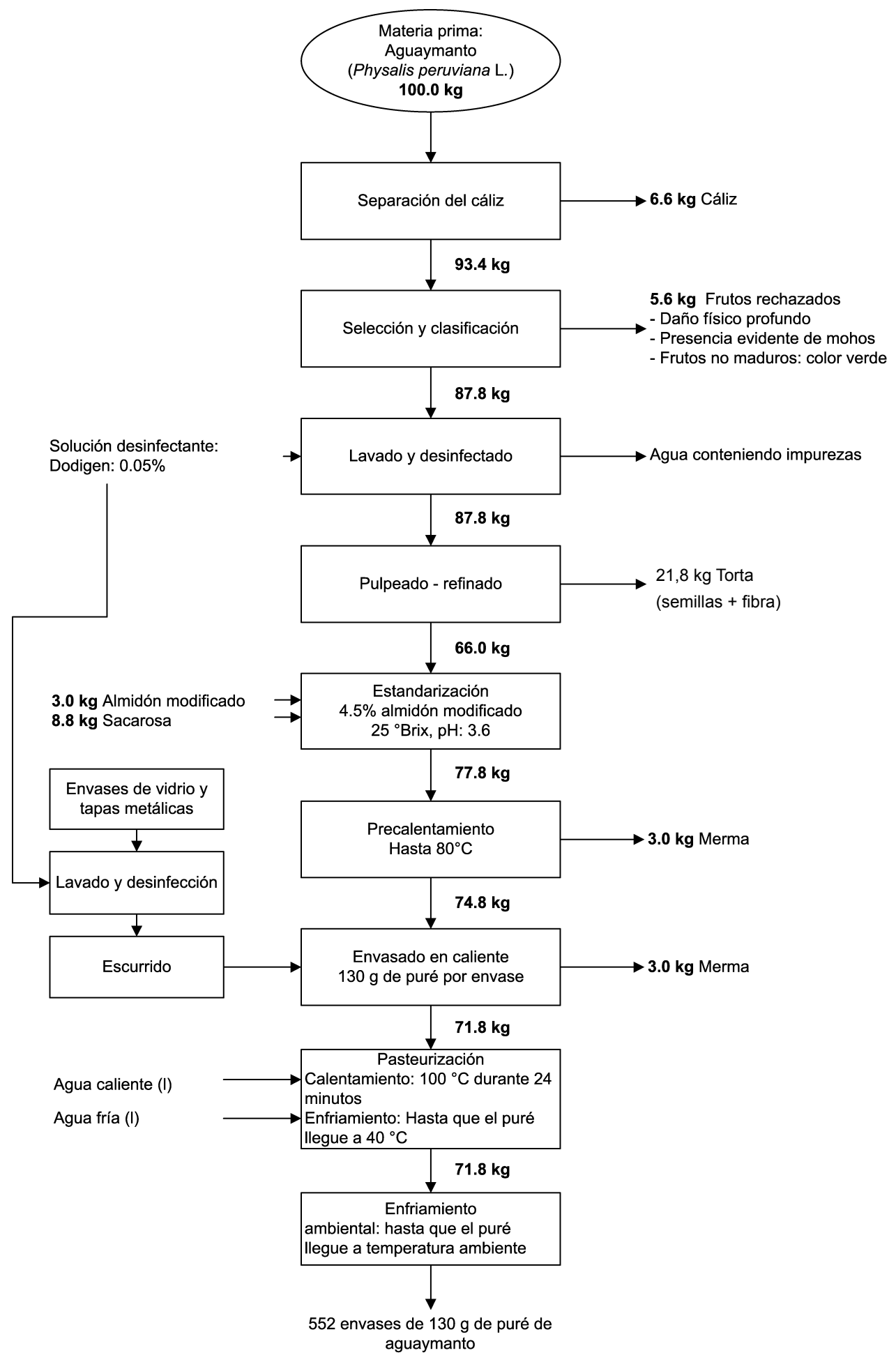

Elaboración propia. 


\subsection{Esquema experimental}

En la figura 2 se muestra el esquema experimental para determinar el proceso y los parámetros de producción del puré de aguaymanto, que se describe en los siguientes incisos.

\subsubsection{Materia prima}

Los frutos de aguaymanto fueron seleccionados y clasificados según su índice de madurez, tamaño, composición proximal, color, sólidos solubles, acidez, $\mathrm{pH}$, azúcares reductores, compuestos bioactivos y capacidad antioxidante.

\subsubsection{Estandarización}

Con la finalidad de obtener un puré de textura cremosa, se utilizó almidón modificado de papa (fosfato de dialmidón hidroxipropilado), el cual es soluble en frío, lo que facilitó la homogeneización. Este aditivo gelatiniza lentamente en la pasteurización, durante la cual el puré permanece líquido, favoreciendo la transferencia de calor por convección para originar, después del enfriamiento, una textura (cremosidad) adecuada y estabilizar el producto al evitar la retrogradación. Fennema (2010) indica que el hidroxipropilalmidón forma pastas claras que no sufren retrogradación, siendo un buen espesante utilizado para mejorar la viscosidad, en particular en condiciones ácidas. Está formado por gránulos reforzados que exhiben una sensibilidad reducida a las condiciones de procesado, como la alta temperatura por tiempos largos y las fuerzas de cizalla.

Se elaboraron muestras de puré de aguaymanto con $\mathrm{pH}$ natural $(3,6)$, utilizando de $2 \%$ a $6 \%$ de almidón (rango recomendado por el fabricante). Dichas muestras fueron pasteurizadas y comparadas visualmente, descartando las que presentaron textura muy fluida o muy viscosa, en comparación con el puré comercial de manzana de marca Gerber ("testigo»); se seleccionó la mejor muestra. Con el objetivo de mejorar la viscosidad, se elaboraron 3 muestras, una con el porcentaje de almidón seleccionado y dos con variaciones de $\pm 0,5 \%$. Dichas muestras, junto con el puré testigo, fueron evaluadas en su viscosidad (Cp) y se seleccionó el rango de concentraciones que se aproximaron más a la viscosidad del puré testigo. 


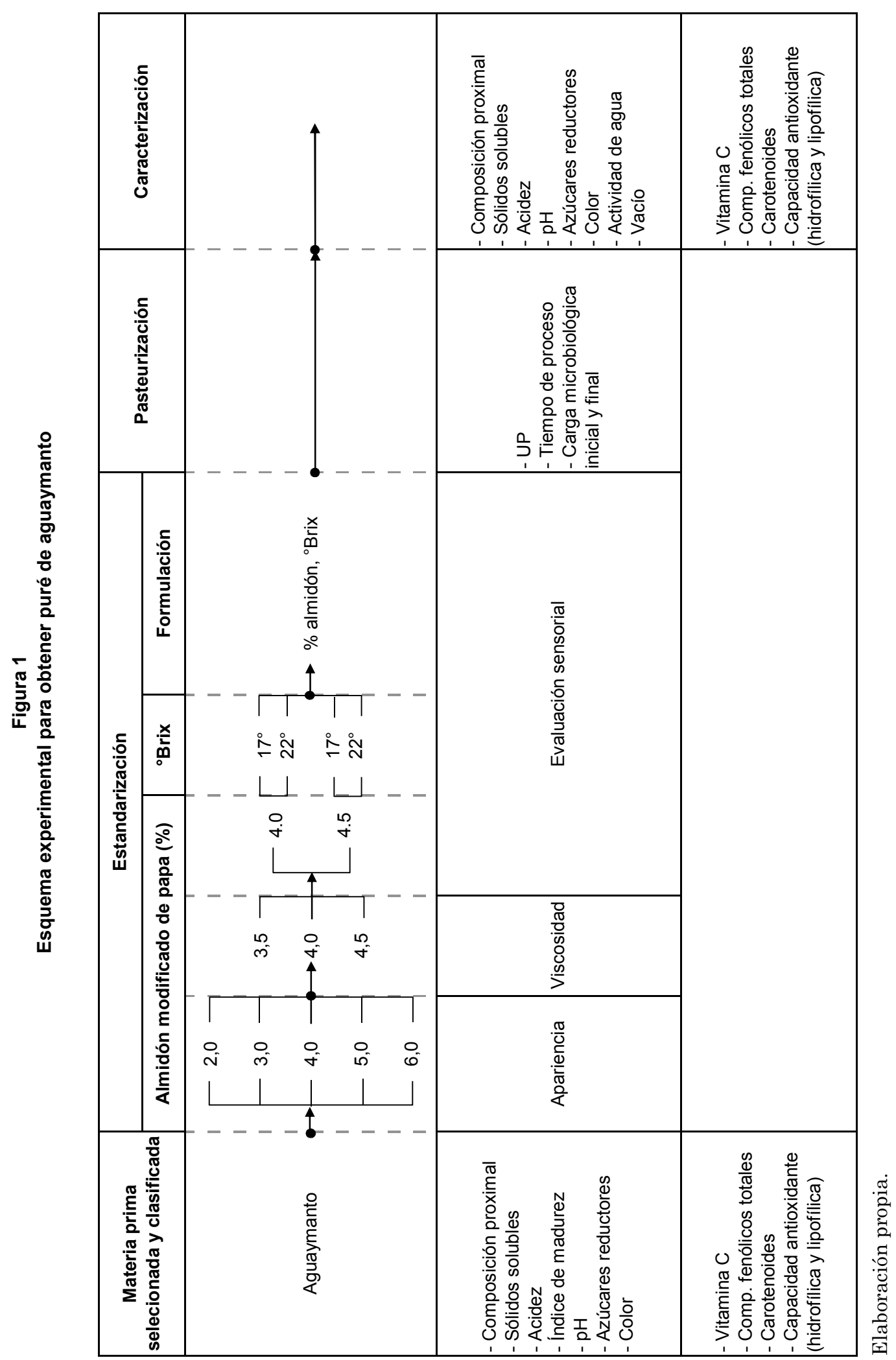

Ingeniería Industrial n. ${ }^{\circ}$ 31, 2013 
Posteriormente, se formularon muestras de puré de aguaymanto con dos niveles de almidón modificado (rango seleccionado) y dos niveles de sólidos solubles agregando azúcar: $17^{\circ}$ Brix (16.5 ${ }^{\circ}$ Brix: valor mínimo según Coddex Alimentarius (1981), STAN 17, para puré de manzanas endulzado en conserva) y $22^{\circ} \mathrm{Brix}$ (valor del colado de frutas tropicales Heinz). De las 4 muestras se seleccionó la muestra que obtuvo el mayor puntaje en una prueba sensorial afectiva de medición del grado de satisfacción (etapa 1), según el procedimiento que se indica en el inciso 2.3.3.

Por último, teniendo en cuenta que en la etapa anterior la apreciación del panel sensorial se inclinó por un mayor dulzor, se elaboró una muestra con $25^{\circ}$ Brix (manteniendo el porcentaje de almidón de la formulación seleccionada en la primera etapa) y se sometió a una evaluación sensorial (etapa 2), cuyo resultado fue comparado con el de la mejor muestra seleccionada en la primera etapa, para determinar la influencia del dulzor en la aceptación del puré de aguaymanto.

\subsubsection{Tratamiento térmico: pasteurización}

Se utilizó el programa Data Trace Temp System ${ }^{\circledR}$ para monitorear el calentamiento a la temperatura recomendada para pasteurización, aproximadamente $100{ }^{\circ} \mathrm{C}$ (agua en ebullición), y enfriamiento del puré de aguaymanto envasado. El monitoreo se realizó en tres puntos equidistantes dentro del envase: a $1 / 4$ de distancia desde la base en el centro geométrico y a $3 / 4$ de distancia desde la base. Con los datos obtenidos de la cinética de calentamiento y enfriamiento se calcularon las velocidades fh y fc, a partir de las cuales se determinó el punto más frío.

La aplicación de $100{ }^{\circ} \mathrm{C}$ en la pasteurización se justificó por ser el puré de aguaymanto un producto altamente ácido; al respecto, Stumbo (1973) indica que el rango de $\mathrm{pH} \leq 3,7$ corresponde a alimentos altamente ácidos. Pokorny, Yanishlieva y Gordon (2001) refieren que la aplicación de temperaturas moderadas $\left(100^{\circ} \mathrm{C}\right.$ como máximo) reduce los cambios negativos que se producen en la calidad nutritiva del alimento. Asimismo, Brennan, Butters y Cowell (1998) refieren que para productos ácidos, como frutas (pH 3,7 a 4,5), se utilizan procesos de tratamiento a $100{ }^{\circ} \mathrm{C}$. Según Rees y Bettinson (1994), cuando el pH es inferior a 3,7, el tratamiento debe orientarse hacia el control de bacterias no esporuladas, levaduras y mohos, pudiendo estos ser controlados con temperaturas incluso inferiores a $100{ }^{\circ} \mathrm{C}$. Hull (1939) indica que 
las ascosporas del moho Byssochlamys fulva mueren a partir de $90^{\circ} \mathrm{C}$, pero algunas podrían sobrevivir hasta temperaturas de $96^{\circ} \mathrm{C}$.

Se establecieron las unidades de pasteurización (UP) necesarias para producir un determinado número de reducciones decimales de la población microbiana inicial (bacterias ácido-lácticas, mohos y levaduras) y llegar a un nivel aceptable de riesgo asumible de encontrar un producto contaminado por cada 1.000.000. Se tomó como referencia el Bacillus coagulans (bacteria ácido-láctica) y el Byssochlamys fulva (moho de mayor termorresistencia). Según Rees y Bettinson (1994), Byssochlamys fulva es el moho que con mayor frecuencia origina alteraciones en productos ácidos conservados por el calor. Stumbo (1973) y Frazier (1981) indican que el Bissochlamys fulva es uno de los microorganismos más termorresistentes en alimentos ácidos $(\mathrm{pH}<4,0)$, que fermenta la pectina y cuyas ascosporas pueden resistir tratamientos térmicos y causar alteraciones en alimentos envasados.

Para establecer el tiempo de proceso se realizaron tres repeticiones del tratamiento térmico, colocando en cada una de ellas los sensores en el puré de aguaymanto envasado (por duplicado) y adicionalmente un tercer sensor en el medio de calentamiento. Se controló en el punto más frío la temperatura del producto y el tiempo en el que se acumuló la letalidad deseada (UP), considerándose como inicio del tiempo de proceso desde que la temperatura del medio de calentamiento llegó a $98 \pm 1{ }^{\circ} \mathrm{C}$. El enfriamiento fue realizado con agua fría corriente hasta que la temperatura del producto estuvo por debajo de los $40{ }^{\circ} \mathrm{C}$.

Los datos generados se evaluaron mediante el método general o gráfico indicado por Simpson, verificando el cumplimiento de las UP en el tiempo de proceso dado. Los resultados fueron corroborados con los datos generados por el programa Data Trace Temp System ${ }^{\circledR}$. Asimismo, se calcularon los factores de las curvas de calentamiento y enfriamiento mediante los métodos matemáticos Stumbo y Hayakawa.

Con el fin de validar el tratamiento térmico, se analizó el contenido de bacterias ácido-lácticas, mohos y levaduras en el puré de aguaymanto antes y después del tratamiento.

\subsubsection{Caracterización del producto final}

Se caracterizó el puré de aguaymanto mediante el análisis de su composición proximal, el color, los sólidos solubles, la acidez, el pH, los 
azúcares reductores, la actividad de agua y el vacío. Asimismo se analizaron sus compuestos bioactivos y su capacidad antioxidante.

El puré de aguaymanto es un producto nuevo; por ello paralelamente se evaluaron productos similares que se comercializan en el mercado local, como el colado de zanahoria, la naranja Heinz y el puré de manzana Gerber, con el objetivo de tener valores de referencia de todos los elementos de composición, así como de los compuestos bioactivos y la capacidad antioxidante.

Adicionalmente, fueron tomadas como referencia la norma general del Codex para confituras, jaleas y mermeladas, Codex Stan 2962009, donde se dan definiciones de un puré a base de frutas, y la norma general del Codex para zumos (jugos) y néctares de frutas, Codex Stan 247-2005, donde se mencionan las técnicas de obtención de un producto tipo puré.

\section{RESULTADOS Y DISCUSIÓN}

\subsection{Caracterización fisicoquímica del aguaymanto}

Los resultados del análisis proximal del fruto concuerdan con los encontrados por Encina (2006), Castro, Rodríguez y Vargas (2008) y Puente et al. (2011).

Respecto al color del fruto, se obtuvieron como promedio los siguientes valores: (anaranjado) $\mathrm{L}^{*}=57,82 \pm 2,94, \mathrm{a}^{*}=15,82 \pm 1,18 \mathrm{y}$ $\mathrm{b}^{*}=53,11 \pm 2,53$. Puente et al. (2011) reportan los rangos de color para el fruto fresco de aguaymanto $L^{*}(70,31$ a 71,37$)$, a* $(14,31$ a 15,20$)$ y $b^{*}(60,84$ a 61,76$)$. Encina (2006) reporta $L^{*}=61,42, a^{*}=10,08$ y b* $=$ 36,52 (índice de madurez 5,5).

La diferencia entre los valores encontrados y los reportados por los autores indicados se atribuyó al estado de madurez de los frutos, especificada en la NTC 4580, por Icontec (1999).

Se obtuvo $24,21 \pm 0,81 \mathrm{mg} / 100 \mathrm{~g}$ b.h. $(131,10 \pm 4,38 \mathrm{mg} / 100 \mathrm{~g}$ b.s. $) \mathrm{de}$ vitamina C, menor a $28,55 \mathrm{mg} / 100 \mathrm{~g}$ b.h., reportado por Encina (2006); se encuentra dentro del rango 20 a $43 \mathrm{mg} / 100 \mathrm{~g}$ b.h. (Puente et al. 2011). Las variaciones son atribuidas al estado de madurez y el tiempo transcurrido entre la cosecha y el momento del análisis. 
En cuanto a compuestos fenólicos totales del fruto (mg AGE/100 g) se obtuvo $58,60 \pm 1,96$ (317,28 10,63 b.s.) dentro del rango de 39,15 a 40,45 y 87 (b.h.), reportado por Puente et al. (2011) y Vasco et al. (2008), respectivamente.

Se obtuvo un contenido de carotenoides totales del fruto $(\mathrm{mg}$ B-caroteno eq./ $100 \mathrm{~g})$ de $2,94 \pm 0,43$ b.h. (15,93 $\pm 2,31$ b.s.), cercano a 2,64 b.h. (Perú), reportado por Repo y Encina (2008). Dutta, Chaudhuri y Chakraborty (2005) indican que las diferencias en composición en cuanto a carotenoides se deben a factores como la variedad, el estado de madurez, el clima (la luz solar incrementa la producción de carotenoides en la planta), la geografía del lugar de producción y las prácticas agrícolas (el uso de ciertos herbicidas afectarían negativamente).

El fruto fresco de aguaymanto tiene una capacidad antioxidante ABTS ( $\mu$ mol Trolox eq./ g) hidrofílica de $3,62 \pm 0,14$ b.h. (19,56 $\pm 0,78$ b.s.) y lipofílica de $0,50 \pm 0,06$ b.h. (2,73 $\pm 0,35$ b.s.), siendo la capacidad antioxidante total, $4,12 \pm 0,18$ b.h. (22,29 $\pm 1,00$ b.s.), valor que está dentro del rango 3,89 y 4,44 b.h., encontrado por Repo y Encina (2008) para índices de madurez de 5 y 6 , respectivamente.

En el cuadro 1 se presentan los resultados de la caracterización fisicoquímica del fruto fresco. Adicionalmente se muestra la composición proximal de la pulpa.

Cuadro 1

Composición fisicoquímica del fruto y la pulpa de aguaymanto

\begin{tabular}{lcccc}
\hline Análisis & \multicolumn{2}{c}{ Fruto fresco } & \multicolumn{2}{c}{ Pulpa } \\
\hline Proximal & *b. húmeda & b. seca & *b. húmeda & b. seca \\
Humedad $(\%)$ & 81,53 & - & 85,80 & - \\
Cenizas totales $(\mathrm{g} / 100 \mathrm{~g})$ & 1,17 & 6,33 & 0,90 & 6,34 \\
Grasa cruda $(\mathrm{g} / 100 \mathrm{~g})$ & 0,26 & 1,41 & 0,00 & 0,00 \\
Proteína cruda $(\mathrm{g} / 100 \mathrm{~g})$ & 1,71 & 9,26 & 0,70 & 4,93 \\
Fibra cruda $(\mathrm{g} / 100 \mathrm{~g})$ & 4,37 & 23,66 & 0,00 & 0,00 \\
Carbohidratos $(\mathrm{g} / 100 \mathrm{~g})$ & 15,33 & 83,00 & 12,60 & 88,73 \\
Otros & & & & \\
Energía total $(\mathrm{Kcal} / 100 \mathrm{~g})$ & 70,50 & 381,70 & 53,20 & 374,65 \\
Sólidos solubles $\left({ }^{\circ} \mathrm{Brix}\right)$ & 13,70 & - & - & - \\
\hline
\end{tabular}


(continuación)

\begin{tabular}{|c|c|c|c|c|}
\hline \multirow{2}{*}{$\begin{array}{l}\text { Análisis } \\
\text { Acidez (g ácido cítrico/ } 100 \text { g) }\end{array}$} & \multicolumn{2}{|c|}{ Fruto fresco } & \multicolumn{2}{|c|}{ Pulpa } \\
\hline & 1,59 & 8,61 & - & - \\
\hline $\begin{array}{l}\text { Índice de madurez } \\
\text { ('Brix/\% ác. cítrico) }\end{array}$ & 8,62 & - & - & - \\
\hline $\mathrm{pH}$ & 3,58 & - & - & - \\
\hline Azúcares reductores (g/ $100 \mathrm{~g})$ & 4,96 & 26,85 & - & - \\
\hline \multicolumn{5}{|l|}{ Compuestos bioactivos (mg/ $100 \mathrm{~g}$ ) } \\
\hline Vitamina C (ácido ascórbico) & 24,21 & 131,10 & - & - \\
\hline $\begin{array}{l}\text { Compuestos fenólicos totales } \\
\text { (AGE: ácido gálico equivalente) }\end{array}$ & 58,60 & 317,28 & - & - \\
\hline $\begin{array}{l}\text { Carotenoides totales } \\
\text { ( } \beta \text {-caroteno eq.) }\end{array}$ & 2,94 & 15,93 & - & - \\
\hline \multicolumn{5}{|l|}{$\begin{array}{l}\text { Capacidad antioxidante } \\
(\mu \mathrm{mol} \text { Trolox equivalente/ g) }\end{array}$} \\
\hline Hidrofílica & 3,62 & 19,56 & - & - \\
\hline Lipofilica & 0,50 & 2,73 & - & - \\
\hline Total & 4,12 & 22,29 & - & - \\
\hline
\end{tabular}

*Análisis realizado por duplicado.

Elaboración propia.

\subsection{Pulpeado-refinado}

El rendimiento obtenido de los frutos frescos con cáliz fue de $88 \pm 3 \%$ de frutos pelados y seleccionados y $66 \pm 4 \%$ de pulpa; este último podría incrementarse de acuerdo con la eficiencia del equipo utilizado.

\subsection{Estandarización}

De las muestras de puré de aguaymanto, preparadas con $2 \%$ a $6 \%$ de almidón modificado de papa (fosfato de dialmidón hidroxipropilado), se seleccionó la que contenía 4,0\% y se descartaron las preparadas con $2 \%$ y $3 \%$ por presentar una textura muy fluida, asimismo se descartaron las que contenían $5 \%$ y $6 \%$ por ser muy viscosas, en comparación con el puré de manzana Gerber («testigo»), que, en el momento de la investi- 
gación, fue el único producto tipo puré a base de fruta con una presentación similar en tamaño y tipo de envase, y sometido a tratamiento térmico para su conservación.

En el cuadro 2 se muestran los resultados de la evaluación de la viscosidad del puré de aguaymanto con tres concentraciones de almidón modificado $3,5 \%, 4,0 \%$ y 4,5\%, además del puré de manzana Gerber como testigo. Como se aprecia, las muestras de puré de aguaymanto cuyas concentraciones de almidón estuvieron entre 4,0\% y 4,5\%, reportaron viscosidades de $2.918 \pm 285$ y $4.797 \pm 169 \mathrm{Cp}$, respectivamente, rango en el que se encuentra la viscosidad del puré de manzana Gerber, $3.492 \pm 125 \mathrm{Cp}$.

Cuadro 2

Viscosidad del puré de aguaymanto con diferentes concentraciones de almidón modificado de papa en comparación con un puré comercial

\begin{tabular}{lc}
\hline Muestra & *Viscosidad (Cp) a $45^{\circ} \mathbf{C}$ \\
\hline Puré de aguaymanto con 3,5\% almidón modificado & $1.560 \pm 141$ \\
Puré de aguaymanto con 4,0\% almidón modificado & $2.918 \pm 285$ \\
Puré de aguaymanto con 4,5\% almidón modificado & $4.797 \pm 169$ \\
Puré de manzana Gerber "testigo" & $3.492 \pm 125$ \\
\hline
\end{tabular}

* Análisis realizado por duplicado.

Elaboración propia.

En el cuadro 3 se presentan los resultados de la evaluación sensorial (etapa 1) de las muestras de puré de aguaymanto con 4,0 y $4,5 \%$ de almidón y sacarosa añadida hasta 17 y $22^{\circ}$ Brix. Se determinó que no existen diferencias estadísticas significativas $(\mathrm{p}<0,05)$ entre los tratamientos $1\left(4,0 \%, 17^{\circ}\right.$ Brix) y $3\left(4,5 \%, 17^{\circ}\right.$ Brix) y entre $2\left(4,0 \%, 22^{\circ}\right.$ Brix $)$ y $4\left(4,5 \%, 22^{\circ}\right.$ Brix). Las letras «a» y «b» representan grupos homogéneos, sin diferencias estadísticamente significativas entre aquellos tratamientos que comparten una misma letra.

De estos resultados, se decidió utilizar 4,5\% de almidón, dado que las muestras presentaron textura más cremosa y consistente, y 22 ${ }^{\circ}$ Brix, porque el grado de satisfacción fue influido por el dulzor; las muestras 2 y 4 con $22^{\circ}$ Brix obtuvieron un puntaje promedio de 5 («me 
gusta ligeramente»), en la escala de 1 a 7 , mientras que las muestras 1 y 3 con $17{ }^{\circ}$ Brix, obtuvieron un puntaje promedio de 4,5 , correspondiendo el valor de 4 a «ni me gusta ni me disgusta».

Una muestra de puré de aguaymanto elaborada con 4,5\% de almidón y $25^{\circ}$ Brix, al ser evaluada sensorialmente (etapa 2), obtuvo un puntaje de 6, equivalente a "me gusta mucho». Según los comentarios recibidos por parte de los jueces, los resultados obtenidos en el análisis sensorial fueron influidos por propiedades sensoriales del aguaymanto, como sabor intenso, elevada acidez y aromaticidad.

Cuadro 3

Comparación sensorial entre tratamientos

\begin{tabular}{ccccc}
\hline Tratamientos & $\begin{array}{c}\text { Almidón } \\
\text { modificado } \\
(\%)\end{array}$ & $\begin{array}{c}\text { Sólidos } \\
\text { solubles } \\
\left({ }^{\circ} \text { Brix }\right)\end{array}$ & $\begin{array}{c}\text { Diferencia } \\
\text { significativa } \\
(\mathbf{p}<\mathbf{0 , 0 5 )}\end{array}$ \\
\hline 1 & 4,0 & 17 & $\mathrm{a}$ & $\mathrm{b}$ \\
2 & 4,0 & 22 & & $\mathrm{~b}$ \\
3 & 4,5 & 17 & $\mathrm{a}$ & $\mathrm{b}$ \\
\hline
\end{tabular}

Elaboración propia.

\subsection{Tratamiento térmico: pasteurización}

\subsubsection{Determinación del punto más frío}

En el cuadro 4 se presentan los resultados de los controles realizados a diferentes alturas dentro del envase. Se encontró que el punto más frío del envase utilizado está ubicado a 1/4 de altura desde la base, ya que la velocidad de calentamiento fh es menor en dicho punto. En la figura 2 se muestra la curva logarítmica de penetración de calor y la proyección de su sección lineal, de la cual se obtuvo la pendiente $1 / \mathrm{fh}$ y el intercepto log [jh(TR-To)], donde TR es la temperatura de procesamiento $\left({ }^{\circ} \mathrm{C}\right)$, To es la temperatura inicial del producto $\left({ }^{\circ} \mathrm{C}\right)$ y jh el factor de retraso en el calentamiento, cuyo valor resulta de la diferencia entre la intersección aparente y la verdadera, (TR - Tpi) - (TR - To). 


\section{Cuadro 4}

Determinación del punto más frío durante el calentamiento

\begin{tabular}{lccc}
\hline Secciones del envase evaluadas & $\begin{array}{c}\mathbf{3 / 4} \text { altura } \\
\text { desde la base }\end{array}$ & Centro & $\begin{array}{c}\mathbf{1 / 4} \text { altura } \\
\text { desde la base }\end{array}$ \\
\hline Pendiente: $1 / \mathrm{fh}$ & $-0,0320$ & $-0,0328$ & $-0,0334$ \\
Intercepto: log [jh(TR-To)] & 1,5878 & 1,7523 & 1,7851 \\
Factor de retraso: jh & 0,9628 & 1,5836 & 2,0530 \\
$\begin{array}{l}\text { Velocidad de calentamiento: fh } \\
\text { (minutos/ ciclo log) }\end{array}$ & 31,2797 & 30,5197 & 29,8998 \\
Temp. pseudo inicial: Tpi $\left({ }^{\circ} \mathrm{C}\right)$ & 61,2943 & 43,4662 & 39,0270 \\
\hline
\end{tabular}

Prueba realizada por duplicado.

Elaboración propia.

Figura 2

Proyección de la sección lineal de la curva logarítmica de penetración de calor en el punto ubicado a $1 / 4$ de altura desde la base

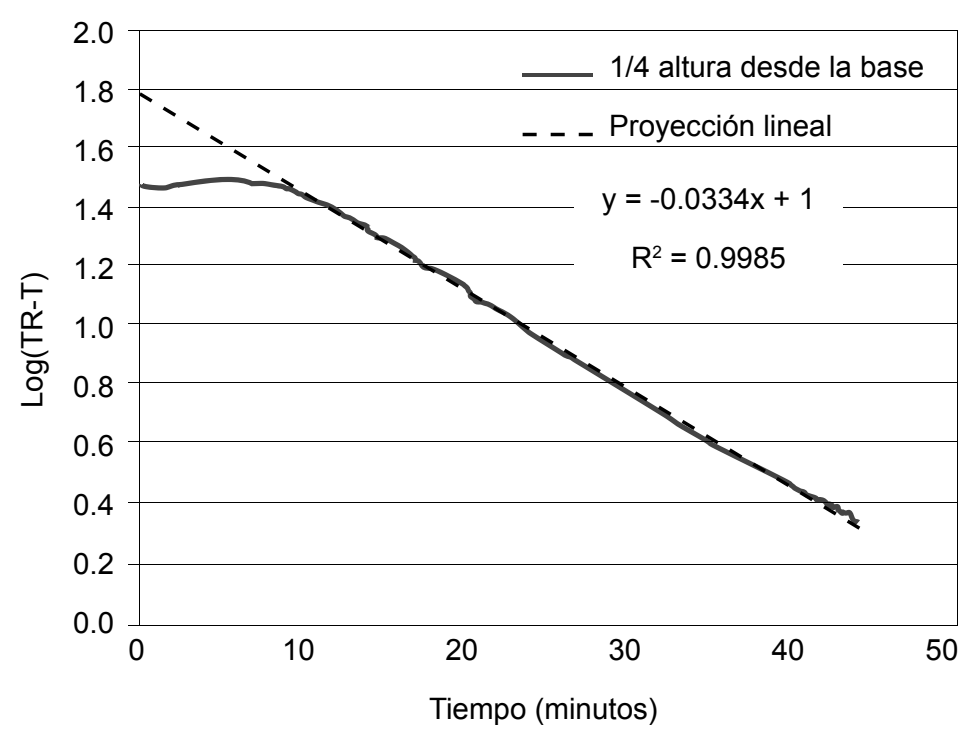

Elaboración propia. 
Fellows (1994) indica que en los envases cilíndricos de aquellos alimentos que se calientan por convección, el punto más frío se encuentra situado en el eje geométrico longitudinal, a un tercio de su altura; el mismo autor señala además que en los alimentos calentados por convección, la situación exacta de este punto varía de acuerdo con el alimento y debe determinarse experimentalmente. Sharma, Mulvaney y Rizvi (2003) señalan que en los alimentos que se calientan por convección y que no se agitan el punto podría localizarse un poco abajo del centro geométrico. Casp y Abril (1999) indican que en productos que se calientan por convección en envases cilíndricos el punto más frío se sitúa en el eje longitudinal, a 1/5 de la altura desde la base.

\subsubsection{Determinación de las unidades de pasteurización UP}

En la evaluación microbiológica de la pulpa de aguaymanto estandarizada, no se detectó la presencia de bacterias ácido-lácticas ( $<10$ UFC/ g), pero sí se encontraron $21.190 \mathrm{UFC/g}$ de mohos y levaduras, equivalente a $2.754 .700 \mathrm{UFC} / 130 \mathrm{~g}$. Se estableció que se necesitan 12 reducciones decimales de la carga inicial, con lo que se tendría una probabilidad de supervivencia microbiana en 1 de cada 1.000.000 unidades de producto, como parámetro de riesgo asumible. Se determinó el tratamiento con el que se obtendría, considerando un margen de seguridad, el grado establecido de destrucción microbiana, $\mathrm{UP}_{100^{\circ} \mathrm{C}}=3,2$ minutos.

\subsubsection{Determinación de los factores de las curvas de calentamiento y enfriamiento}

Las proyecciones lineales de las curvas de calentamiento y enfriamiento arrojaron coeficientes de $\mathrm{R}^{2}=99,74$ y 99,85 , respectivamente.

En el cuadro 5 se muestran los factores calculados a partir de las curvas de calentamiento y enfriamiento. Se encontró una diferencia de 6,65 minutos entre fh y fc, lo que indicaría que la transferencia de calor es una combinación de convección y conducción; al respecto, Stumbo (1973) indica que fh y fc se igualarían al tratarse de convección o conducción pura. El mismo autor hace referencia a que si la diferencia es mayor al $20 \%$, debe utilizarse el método gráfico para evaluar el proceso. En esta investigación se obtuvieron valores de UP y tiempo de proceso, muy cercanos con los tres métodos empleados. 


\section{Cuadro 5}

Factores de las curvas de calentamiento y enfriamiento

\begin{tabular}{|c|c|}
\hline Factores & *Promedio \\
\hline \multicolumn{2}{|l|}{ Calentamiento } \\
\hline Velocidad de calentamiento: fh (minutos) & $28,99 \pm 2,15$ \\
\hline Temperatura inicial: $\mathrm{Tih}=\mathrm{To}\left({ }^{\circ} \mathrm{C}\right)$ & $65,23 \pm 0,35$ \\
\hline $\mathrm{Ih}=\mathrm{TR}-\mathrm{Tih}\left({ }^{\circ} \mathrm{C}\right)$ & $34,77 \pm 0,35$ \\
\hline jhlh = TR $-\mathrm{Tpih}\left({ }^{\circ} \mathrm{C}\right)$ & $36,66 \pm 8,13$ \\
\hline Temperatura seudoinicial: $\operatorname{Tpih}\left({ }^{\circ} \mathrm{C}\right)$ & $63,34 \pm 8,13$ \\
\hline Factor de retraso: jh & $1,05 \pm 0,22$ \\
\hline \multicolumn{2}{|l|}{ Enfriamiento } \\
\hline Velocidad de enfriamiento: fc (minutos) & $35,64 \pm 2,97$ \\
\hline Temperatura inicial: $\operatorname{Tic}=\operatorname{Tg}\left({ }^{\circ} \mathrm{C}\right)$ & $95,87 \pm 0,38$ \\
\hline $\mathrm{Ic}=\mathrm{Tg}-\mathrm{Tc}\left({ }^{\circ} \mathrm{C}\right)$ & $75,87 \pm 0,38$ \\
\hline $\mathrm{jclc}=\mathrm{Tpic}-\mathrm{Tc}\left({ }^{\circ} \mathrm{C}\right)$ & $86,32 \pm 8,43$ \\
\hline Temperatura seudoinicial: Tpic $\left({ }^{\circ} \mathrm{C}\right)$ & $106,32 \pm 8,43$ \\
\hline Factor de retraso: Jc & $1,14 \pm 0,12$ \\
\hline
\end{tabular}

*Estudio realizado por triplicado.

Elaboración propia.

En lo que respecta al factor de retraso (j), Stumbo (1973) reporta que en alimentos, donde la transferencia de calor se da por convección pura, $\mathrm{jc}=1$, y en el caso de conducción pura, jc $=2$, según los resultados obtenidos, la transferencia de calor mayoritariamente fue por convección, ya que los valores de jh $=1,05$ y jc $=1,14$ son muy cercanos a 1 . 
$\mathrm{El}$ producto, cuya temperatura inicial fue $65^{\circ} \mathrm{C}$, alcanzó $96^{\circ} \mathrm{C}$ en su punto más frío durante el tratamiento térmico. La temperatura inicial del producto influyó de manera determinante en la velocidad fh, debido al diferencial de temperatura Ih, contribuyendo además a la generación de vacío; sería materia de evaluación la eficiencia de un proceso de exhausting para incrementar dicha temperatura, incrementando a su vez el vacío y reduciendo el tiempo de proceso. En el caso de la velocidad de enfriamiento fc, esta fue mayor que fh, debido a un mayor diferencial de temperaturas Ic, lo que produjo mayor rapidez en el enfriamiento; de esta forma se evitó que el producto estuviera expuesto durante periodos prolongados a temperaturas favorables para el crecimiento microbiano.

La proyección lineal de la curva de penetración de calor produjo parámetros que permiten evaluar el proceso en diferentes condiciones, lo cual permitió simulaciones con diversas aplicaciones. Los parámetros aparentes fueron calculados a partir de temperaturas reales, considerando los factores de retraso $\mathrm{j}$.

\subsubsection{Validación del tratamiento térmico}

Luego de la pasteurización del puré de aguaymanto no se detectó la presencia de mohos, levaduras ni bacterias ácido-lácticas ( $<10 \mathrm{UFC/g})$, cumpliendo así con los límites $\left(10^{2} \mathrm{UFC} / \mathrm{g}\right)$ para productos con $\mathrm{pH}<4,6$ (semiconservas) indicados en la NTS 071-Minsa/Digesa.

\subsection{Caracterización del puré de aguaymanto}

En el cuadro 6 se presentan los resultados de la composición proximal del puré de aguaymanto, el cual contiene aproximadamente $85 \%$ de pulpa, azúcar (hasta lograr $25^{\circ}$ Brix) y 4,5\% de almidón. Asimismo, luego de analizar los compuestos bioactivos y la capacidad antioxidante del puré de aguaymanto, se desprende que el producto obtenido se puede utilizar como un alimento funcional. 


\section{Cuadro 6}

Caracterización fisicoquímica del puré de aguaymanto

\begin{tabular}{|c|c|c|}
\hline \multirow[t]{2}{*}{ Análisis } & \multicolumn{2}{|c|}{ Puré de aguaymanto } \\
\hline & *b.h. & b.s. \\
\hline \multicolumn{3}{|l|}{ Composición proximal } \\
\hline Humedad (\%) & 72,70 & - \\
\hline Cenizas totales $(\mathrm{g} / 100 \mathrm{~g})$ & 0,70 & 2,56 \\
\hline Grasa cruda (g/100 g) & 0,00 & 0,00 \\
\hline Proteína cruda $(\mathrm{g} / 100 \mathrm{~g})$ & 0,70 & 2,56 \\
\hline Fibra cruda (g/100 g) & 0,00 & 0,00 \\
\hline Carbohidratos (g/100 g) & 25,90 & 94,87 \\
\hline \multicolumn{3}{|l|}{ Otros } \\
\hline Energía total $(\mathrm{kcal} / 100 \mathrm{~g})$ & 106,40 & 389,74 \\
\hline Sólidos solubles ( ${ }^{\circ}$ Brix) & 24,9 & - \\
\hline Acidez (g ácido cítrico/100 g) & 1,41 & 5,16 \\
\hline $\mathrm{pH}$ & 3,66 & \\
\hline Azúcares reductores $(\mathrm{g} / 100 \mathrm{~g})$ & 8,17 & 29,93 \\
\hline Color $\left(L^{*}, a^{*}, b^{*}\right)$ & $21,18,2,59,16,12$ & - \\
\hline Vacío (in Hg) & 10 & - \\
\hline Actividad de agua aw & 0,973 & - \\
\hline \multicolumn{3}{|l|}{ Compuestos bioactivos $(\mathrm{mg} / 100 \mathrm{~g})$} \\
\hline Vitamina C & 18,91 & 69,25 \\
\hline Compuestos fenólicos totales (AGE) & 48,93 & 179,22 \\
\hline Carotenoides totales ( $\beta$-caroteno eq.) & 1,70 & 6,25 \\
\hline \multicolumn{3}{|l|}{$\begin{array}{l}\text { Capacidad antioxidante } \\
(\mu \mathrm{mol} \text { Trolox eq./g) }\end{array}$} \\
\hline Hidrofílica & 3,42 & 12,28 \\
\hline Lipofílica & 0,37 & 1,34 \\
\hline Total & 3,78 & 13,62 \\
\hline
\end{tabular}

*Análisis realizado por duplicado.

Elaboración propia. 


\section{Cuadro 7}

Caracterización fisicoquímica de productos del mercado (referencias)

\begin{tabular}{|c|c|c|}
\hline Análisis & $\begin{array}{c}\text { Puré de manzana } \\
\text { Gerber }\end{array}$ & $\begin{array}{c}\text { Colado de zanahoria } \\
\text { y naranja Heinz }\end{array}$ \\
\hline \multicolumn{3}{|l|}{ Composición proximal } \\
\hline Humedad (\%) & - & - \\
\hline Cenizas totales $(\mathrm{g} / 100 \mathrm{~g})$ & - & - \\
\hline Grasa cruda (g/100 g) & $0,0^{(1)}$ & $0,1^{(1)}$ \\
\hline Proteína cruda (g/100 g) & $0,0^{(1)}$ & $0,3^{(1)}$ \\
\hline Fibra cruda (g/100 g) & - & - \\
\hline Carbohidratos (g/100 g) & $17^{(1)}$ & $21,1^{(1)}$ \\
\hline \multicolumn{3}{|l|}{ Otros } \\
\hline Energía total (Kcal/100 g) & $71^{(1)}$ & $86^{(1)}$ \\
\hline Sólidos solubles ( ${ }^{\circ}$ Brix) & $17,0^{(2)}$ & $19,2^{(2)}$ \\
\hline Acidez (g ácido cítrico/100 g) & $1,0^{(2)}$ & $1,4^{(2)}$ \\
\hline $\mathrm{pH}$ & $3,9^{(2)}$ & $3,8^{(2)}$ \\
\hline Azúcares reductores (g/100 g) & $51,5^{(2)}$ & $17,4^{(2)}$ \\
\hline Color $\left(L^{*}, a^{*}, b^{*}\right)$ & - & - \\
\hline Vacío (in Hg) & $22,5^{(2)}$ & $23,5^{(2)}$ \\
\hline Actividad de agua $a_{w}$ & $0,983^{(2)}$ & $0,985^{(2)}$ \\
\hline \multicolumn{3}{|l|}{ Compuestos bioactivos (mg/100 g) } \\
\hline Vitamina C & $9^{(1)}$ & $45^{(1)}$ \\
\hline Compuestos fenólicos totales (AGE) & $71^{(2)}$ & $46^{(2)}$ \\
\hline Carotenoides totales ( $\beta$-caroteno eq.) & - & $2,4^{(2)}$ \\
\hline \multicolumn{3}{|l|}{$\begin{array}{l}\text { Capacidad antioxidante } \\
(\mu \mathrm{mol} \text { Trolox eq./ g) }\end{array}$} \\
\hline Hidrofílica & $4,4^{(2)}$ & $2,9^{(2)}$ \\
\hline Lipofílica & - & $0,2^{(2)}$ \\
\hline Total & $4,4^{(2)}$ & $3,1^{(2)}$ \\
\hline
\end{tabular}

(1) Datos reportados en la etiqueta del producto (b.h.).

(2) Resultados promedio de análisis llevados a cabo durante la investigación

(b.h.). Los análisis fueron realizados por duplicado.

Elaboración propia. 
En el cuadro 7 se muestra la composición de productos del mercado, similares en ciertas características al puré de aguaymanto, que se encontraron en venta en el momento de la investigación. Esta información se utilizó como referencia, ya que el puré de aguaymanto es un producto nuevo.

En cuanto a la composición proximal del puré de aguaymanto, esta es similar a los productos referenciales.

En el puré de aguaymanto se presentó un valor mayor de sólidos solubles, $25^{\circ}$ Brix, producto del dulzor al que tuvo que llegarse para tener una mayor aceptación sensorial. El pH 3,7 es menor, característica propia del fruto, contribuyendo a la conservación del producto. Estándares descritos por la USDA (2000), donde se hace referencia a purés de manzana, plátano, arándano, entre otros, indican rangos de sólidos solubles que van desde 6 hasta $24^{\circ}$ Brix y valores de $\mathrm{pH}$ que van desde 2,8 hasta 4,5 .

El vacío de 10 in $\mathrm{Hg}(254 \mathrm{~mm} \mathrm{Hg})$ del puré de aguaymanto es menor que el de los productos referenciales. Sin embargo, Rees y Bettison (1994) indican que en productos pasteurizados, un nivel de $200 \mathrm{~mm} \mathrm{Hg}$ es suficiente para mantener el vacío en el interior de los recipientes.

En cuanto a los compuestos bioactivos y la capacidad antioxidante, el puré de aguaymanto se encuentra dentro de los rangos de los productos referenciales, con un contenido de carotenoides ligeramente menor.

\section{CONCLUSIONES}

1. Los parámetros de procesamiento para obtener puré de aguayman-

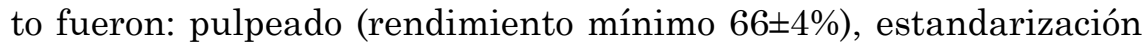
(4,5\% de almidón modificado y sacarosa hasta llegar a $\left.25^{\circ} \mathrm{Brix}\right) \mathrm{y}$ pasteurización $\left(100{ }^{\circ} \mathrm{C}\right.$ por 24 minutos: $\mathrm{UP}=3,2$ minutos). La evaluación sensorial del producto obtenido reportó un puntaje de 6 , equivalente a "me gusta mucho» en la escala predeterminada.

2. La composición fisicoquímica del fruto de aguaymanto fue por cada $100 \mathrm{~g}$ b.s.: humedad 81,53\% (b.h.), cenizas $6,33 \mathrm{~g}$, grasa $1,41 \mathrm{~g}$, proteína 9,26 g, fibra cruda 23,66 g, carbohidratos $83,00 \mathrm{~g}$, energía $381,70 \mathrm{Kcal}$, acidez $8,61 \pm 0,70 \mathrm{~g}$, ácido cítrico y azúcares reductores $26,85 \pm 2,44 \mathrm{~g}$, caracterizándose por tener un índice de madurez de 8,6 (color 5 a 6 ), sólidos solubles $13,7 \pm 0,6^{\circ} \mathrm{Brix}$, $\mathrm{pH} 3,58 \pm 0,15$ y color $\left(\mathrm{L}^{*}=57,82 \pm 2,94, \mathrm{a}^{*}=15,82 \pm 1,18 \mathrm{y} \mathrm{b}^{*}=53,11 \pm 2,53\right)$. El contenido de 
compuestos bioactivos del aguaymanto fue de ácido ascórbico (b.s) $131,10 \pm 4,38 \mathrm{mg} / 100 \mathrm{~g}$, compuestos fenólicos totales (b.s.), 317,28 $\pm 10,63 \mathrm{mg} \mathrm{AGE} / 100 \mathrm{~g}$, carotenoides totales (b.s.), 15,93 $\pm 2,31 \mathrm{mg}$ B-caroteno eq./100 g. La capacidad antioxidante ( $\mu$ mol Trolox eq./ g b.s.) hidrofílica fue de $19,56 \pm 0,78$, la capacidad antioxidante lipofílica fue $2,73 \pm 0,35$, sumando una capacidad antioxidante total de $22,29 \pm 1,00$.

3. La composición fisicoquímica del puré de aguaymanto fue por cada 100 g b.s.: humedad 72,70 \% (b.h.), cenizas 2,56 g, proteína 2,56 g, carbohidratos $94,87 \mathrm{~g}$, energía $389,74 \mathrm{Kcal}$, grasa y fibra cruda

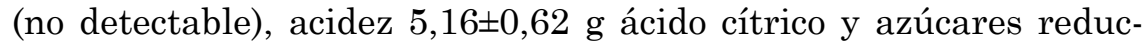
tores $29,93 \pm 3,77 \mathrm{~g}$, caracterizándose por tener un $\mathrm{pH}$ de 3,66 $\pm 0,14$, color $\left(\mathrm{L}^{*}=21,18 \pm 4,06, \mathrm{a}^{*}=2,59 \pm 1,19 \mathrm{y} \mathrm{b}^{*}=16,12 \pm 5,90\right)$ y una $\mathrm{a}_{\mathrm{w}} \mathrm{de}$ $0,973 \pm 0,001$. El contenido de compuestos bioactivos del puré de aguaymanto fue de ácido ascórbico (b.s) 69,25 $\pm 1,30 \mathrm{mg} / 100 \mathrm{~g}$, compuestos fenólicos totales (b.s.), 179,22 $\pm 11,94 \mathrm{mg}$ AGE/100 g, carotenoides totales (b.s.), 6,25 $\pm 1,44 \mathrm{mg}$ B-caroteno eq./100 g. La capacidad antioxidante ( $\mathrm{mmol}$ Trolox eq./ g b.s.) hidrofílica fue de 12,28 $\pm 0,73$, la capacidad antioxidante lipofílica fue $1,34 \pm 0,14$, sumando una capacidad antioxidante total de 13,62 $\pm 0,75$.

\section{RECOMENDACIONES}

1. Evaluar la mezcla del aguaymanto con otros frutos para diversificar las características sensoriales del puré, así como la aplicación de diluciones de las pulpas que se van a utilizar.

2. Experimentar el reemplazo de la sacarosa, por edulcorantes naturales, determinando su impacto en la calidad sensorial y fisicoquímica del producto.

3. Realizar estudios para la caracterización reológica del puré de aguaymanto.

4. Evaluar el uso de un pasteurizador tubular de superficie rascada para determinar el efecto de la pasteurización en los compuestos bioactivos y la capacidad antioxidante.

5. Realizar el estudio de vida en anaquel del puré de aguaymanto.

6. Establecer el uso de los subproductos y determinar sus propiedades. 


\section{GLOSARIO}

$\mathrm{L}^{*}$ : $\quad$ Claridad. Luminosidad del color $\left(\mathrm{L}^{*}=0\right.$ : negro y $\mathrm{L}^{*}=100$ : blanco).

a*: $\quad$ Cromaticidad. Valores negativos (verde) y valores positivos (magenta).

$b^{*}: \quad$ Cromaticidad. Valores negativos (azul) y valores positivos (amarillo).

$\mathrm{a}_{\mathrm{w}}: \quad$ Actividad de agua

in $\mathrm{Hg}$ : $\quad$ Pulgadas de mercurio.

ETA: $\quad$ Enfermedad transmitida por alimentos.

fh: $\quad$ Velocidad de penetración de calor (minutos).

Tih = To: $\quad$ Temperatura inicial del producto al inicio del proceso de calentamiento $\left({ }^{\circ} \mathrm{C}\right)$.

$\mathrm{T}_{\mathrm{R}}: \quad$ Temperatura del medio de calentamiento $\left({ }^{\circ} \mathrm{C}\right)$.

Ih: Diferencia inicial de temperatura: $\mathrm{T}_{\mathrm{R}}-\mathrm{T}_{0}\left({ }^{\circ} \mathrm{C}\right)$

Tpih: $\quad$ Temperatura seudoinicial $\left({ }^{\circ} \mathrm{C}\right)$.

Jh: $\quad$ Factor de retraso. Tiempo que transcurre antes de que la velocidad de penetración de calor alcance fh.

fc: $\quad$ Velocidad de enfriamiento (minutos).

Tic $=$ Tg: $\quad$ Temperatura del producto al inicio del enfriamiento $\left({ }^{\circ} \mathrm{C}\right)$.

Tc: $\quad$ Temperatura del medio de enfriamiento $\left({ }^{\circ} \mathrm{C}\right)$.

Ic: Diferencia inicial de temperatura (en el enfriamiento): $\mathrm{Tg}-\mathrm{Tc}\left({ }^{\circ} \mathrm{C}\right)$.

Tpic: $\quad$ Temperatura seudoinicial de enfriamiento $\left({ }^{\circ} \mathrm{C}\right)$.

Jc: $\quad$ Factor de retraso. Tiempo que transcurre antes de que la velocidad de penetración de calor alcance fc. 


\section{REFERENCIAS}

Anzaldúa-Morales, A. (1994). Evaluación sensorial de los alimentos en la teoría y la práctica. Zaragoza: Acribia.

Araujo, G. E. (2009). El cultivo de aguaymanto o tomatillo (Physalis peruviana L.): manejo técnico en los Andes del Perú. Cultivos andinos. Guía técnica de recopilación bibliográfica. Cajamarca. Recuperado de http://aguaymanto.blog.galeon.com

Arnao, M., Cano, A., \& Acosta, M. (2001). The hidrophilic and lipophilic contribution to total antioxidant activity. Food Chemistry, 73, 239-244. Murcia.

Association of Analytical Communities (2005). Official methods of analysis. (18. ${ }^{\mathrm{a}}$ ed.).

Brennan, J. G., Butters, J. R., \& Cowell, N. D. (1998). Las operaciones de la ingeniería de los alimentos. Zaragoza: Acribia.

Casp, V., Ana, \& Abril R., José (1999). Procesos de conservación de alimentos. Madrid: Mundi-Prensa.

Castro, A., Rodríguez, L., \& Vargas, E. (2008). Secado de uchuva (Physalis peruviana L.) por aire caliente con pretratamiento de osmodeshidratación. Revista de la Facultad de Química Farmacéutica, 15(2), 226-231. Medellín.

Commission Internationale D'eclairage: Comisión Internacional de la Iluminación. (1986). Color measurement. Viena.

Comisión del Codex Alimentarius (2001). Codex Stan 17-1981. Norma del Codex para el puré de manzanas en conserva. Roma.

Comisión del Codex Alimentarius (2005). Codex Stan 247-2005. Norma general del Codex para zumos (jugos) y néctares de frutas. Roma.

Comisión del Codex Alimentarius (2009). Codex Stan 296-2009. Norma general del Codex para confituras, jaleas y mermeladas. Roma.

Collazos, C. (1993). La composición de alimentos de mayor consumo en el Perú. (6. ${ }^{\text {a }}$ ed.). Lima: Ministerio de Salud, Instituto Nacional de Nutrición.

Dirección General de Salud Ambiental (2008). NTS $N^{\circ} 071$ Minsa/ Digesa-V.01: Norma sanitaria que establece los criterios micro- 
biológicos de calidad sanitaria e inocuidad para los alimentos y bebidas de consumo humano. Resolución Ministerial 591 Minsa. Lima.

Dutta, D., Chaudhuri, U. R., \& Chakraborty, R. (2005). Review. Structure, health benefits, antioxidant property and processing and storage of carotenoids. African Journal of Biotechnology, 4(13), 1510-1520. Recuperado de http://www.academicjournals.org/AJB

Encina, C. (2006). Influencia del descerado y composición del almíbar en la optimización del tratamiento térmico de la conserva de aguaymanto (Physalis peruviana Linnaeus, 1753.) para la mayor retención de ácido ascórbico. Tesis para optar el título de magíster. Lima: Universidad Nacional Agraria La Molina.

Espinoza, Atencia E. (2003). Evaluación sensorial de los alimentos. Tacna: Universidad Nacional Jorge Basadre Grohmann.

Fennema, O. (2010). Química de los alimentos. Zaragoza: Acribia.

Fellows, P. (1994). Tecnología del procesado de alimentos: Principios y prácticas. Zaragoza: Acribia.

Frazier, W. C. (1981). Microbiología de los alimentos. Zaragoza: Acribia.

Hull, R. (1939). Study of Byssochlamys fulva and control measures in processed fruits. (2. ${ }^{a}$ ed.). Annals of Applied Biology, 26(4), 800822. Londres.

Instituto Colombiano de Normas Técnicas y Certificación. (1999). Norma Técnica Colombiana NTC 458: Frutas frescas. Uchuva. Especificaciones. Bogotá.

Instituto Nacional de Defensa de la Competencia y de la Protección de la Propiedad Intelectual. NTP 203.121:2007: Frutas andinas tipo berries (bayas). Aguaymanto (Physalis peruviana L.) fresco. Lima.

Instituto Nacional de Defensa de la Competencia y de la Protección de la Propiedad Intelectual. NTP 205.003:1980, Revisión 2011: Cereales y menestras. Determinación de la fibra cruda. Lima.

Instituto Nacional de Defensa de la Competencia y de la Protección de la Propiedad Intelectual. NTP 203.077:1977, Revisión 2012. 
Productos elaborados a partir de frutas y otros vegetales. Determinación del vacío. Lima.

Masure, M. P., \& Cambell, H. (1944). Rapid estimation of peroxidase in vegetable extracts as index of blanching for frozen vegetables. The fruit products. Journal American Food Manufacture, 23(8).

Mouquet, Claire, \& Tréche, Serge (2001). Viscosity of gruels for infants: a comparison of measurement procedures. International Journal of Food Sciences and Nutrition, 52, 389-400.

Muñoz, J. A., Ramos-Escudero, D., Alvarado Ortiz, C., \& Castañeda, C. B. (2007). Evaluación de la capacidad antioxidante y contenido de compuestos fenólicos en recursos vegetales promisorios. Revista Sociedad Química del Perú, 75(3), 142-149. Lima.

Pantelidis, G. E., Vasilakakis, M., Manganaris, G. A., \& Diamantidis, G. (2007). Antioxidant capacity, phenol, anthocyanin and ascorbic acid contents in raspberries, blackberries, red currants, gooseberries and cornelian cherries. Food Chemistry, 102, 777783. Recuperado de http://www.sciencedirect.com

Pokorny, J., Yanishlieva, N., \& Gordon, M. (2001). Antioxidantes de los alimentos. Aplicaciones prácticas. Zaragoza: Acribia.

Puente, L. A., Pinto, C., Castro, E., \& Cortes, M. (2011). Physalis peruviana Linnaeus, the multiple properties of a highly functional fruit: A review. Food Research International, 44, 1733-1740.

Rees, J. A. G., \& Bettinson, J. (1994). Procesado térmico y envasado de alimentos. Zaragoza: Acribia.

Repo de Carrasco, R. \& Encina Zelada, C. (2008). Determinación de la capacidad antioxidante y compuestos bioactivos de frutas nativas peruanas. Revista Sociedad Química del Perú, 74(2), 108-124.

Sharma, Shri, Mulvaney, Steven, \& Rizvi, Syed (2003). Ingeniería de alimentos. Operaciones unitarias y prácticas de laboratorio. $\left(1 .^{\mathrm{a}}\right.$ ed). Nueva York: Limusa.

Sistema Integrado de Información de Comercio Exterior. Exportación del producto aguaymanto según sus principales mercados en $\mathrm{kg}$, período 2007-2012. Recuperado el 20 de noviembre del 2011, de http://www.siicex.gob.pe 
Stumbo, C. R. (1973). Thermobacteriology in food processing. Nueva York y Londres: Academic Press.

Swain, T., \& Hillis, W. E. (1959). The phenolic constituents of Prunus domestica. The quantitative analysis of phenolic constituents. Journal of Science of Food and Agriculture, 10, 63-68.

Talcott, S. T., \& Howard, L. R. (1999). Phenolic autoxidation is responsible for color degradation in processed carrot puree. Journal of Agricultural and Food Chemistry, (5), 2109-2115.

United States Department of Agriculture (USDA) (2000). Commercial item description. fruit purees. Recuperado el 20 de setiembre del 2011, de http://www.ams.usda.gov/AMSv1.0/ getfile?dDocName=STELDEV3006919

Vasco, C., Ruales, J., \& Kamal-Eldin, A. (2008). Total phenolic compounds and antioxidant capacities of major fruits from Ecuador. Food Chemistry, 111(4), 816-823. 